- Not all neural networks require a teacher to acquire interesting properties. At the Neuroscience meeting (see left), Ralph Linsker (IBM Thomas J. Watson Laboratory) explained how feature detectors can emerge from an untrained network, suggesting that in early development of the nervous system, patterned connections may not have to be specified in detail, but can arise naturally from a generic rule.

In Linsker's model ${ }^{6-8}$, cells are arranged in a stack of layers, each cell's output to the layer above being a weighted average of inputs received from an underlying region of cells in the next layer down. Inputs are 'hebbian': they increase their connection strength if activity in the input cell correlates with activity in the output cell. The blind workings of this rule have subtle effects. Even random input causes feature analysers - contrast- and orientationselective cells - to emerge in later layers.

Why does this surprising result emerge? A little thought suggests an answer. Each cell receives inputs from many Hebb synapses; each connection will increase (decrease) its strength when it 'agrees' ('disagrees') with the output. An analogy is that of an election where all those who vote for the winning candidate are listened to more carefully in the next election. Thus, groups that vote the same way emerge if there is any structure in (correlations among) their inputs. Structure can come in the the input from the environment.

Where random inputs are provided to the first layer (analogous to the situation of animals born with their eyes still shut) the wiring provides structure. A cell in the second layer receives more input from cells directly beneath it than from those further away. As connection strengths fluctuate and evolve, the set of units that are central to a particular second-layer cell will come to vote as a block; either as an all excitatory or all inhibitory unit. With a structured input coming from the second layer, further layers will add more structure.

The Hebb rule can be described as producing cells whose output varies as much as possible with variation in input from the previous layer of cells so that they signal the maximum amount of information about what is happening there. This 'infomax' principle, as Linsker calls it, can be made rigorous so that it relates input and output variance in a mathematically definable way.

When this principle is put to work in a simulation, topographic maps can be made to emerge that look identical to those found in the cortex. There is thus the possibility that a part of the order seen in the brain emerges as a result of a general information-processing principle. How exactly this principle could be being executed by real neurons remains to be seen. But if true, there are pleasing implications for evolution - generic principles permit re-routing of information without a complete redesign.

Alun Anderson
Galactic nuclei \section{Stellar ejecta from black holes}

\author{
M.E. Bailey
}

THE resolution of the question of whether there is a massive black hole (at least a million times more massive than the Sun) at the centre of our Galaxy has crucial implications for our understanding of the evolution of galactic nuclei. Although some studies ${ }^{1-5}$ conclude that there is no such central object, others ${ }^{6-8}$ emphasize that the evidence is inconclusive. The prevailing view, however, is that black holes most naturally explain active galactic nuclei and the quasar phenomenon, and most astrophysicists believe that the black-hole hypothesis gives the best overall explanation of the complex picture now emerging from multi-frequency observations of the galactic centre ${ }^{-12}$. On page 687 of this issue ${ }^{13}$, Hills gives an extra twist to the problem, showing that a massive black hole in the galactic centre should be a significant source of 'hypervelocity' stars.

Close binary systems (tightly bound pairs of stars) with orbital velocities $V_{\text {orb }}$ greater than the average stellar velocity dispersion are generally expected to survive in galactic nuclei. Such binaries are usually described as being 'hard', and evolve under external stellar perturba- tions by transferring orbital kinetic energy to the surrounding stars, thus becoming harder and increasingly tightly bound ${ }^{14}$. Hills's work shows, however, that if the star cluster contains a massive central object such as a black hole, the close passage of the binary past the massive body could result in a so-called 'exchange' collision. The three-body interaction of the binary with the black hole results in a net perturbation in which one star is captured into a tightly-bound orbit about the black hole, and the other is ejected at high speed into the surrounding galaxy.

The effect is entirely newtonian, and can be approximately understood in terms of the interaction between the two binary components during the period of closest approach to the black hole. (The situation is analogous, in this respect, to the randomizing effects of external stellar perturbations on the orbital energies of binary stars or of comets in the Oort cloud.) Hence, if one component of the binary (of semi-major axis $a$ and total mass about that of the Sun, $1 M_{\odot}$ ) is regarded as being in an orbit about the black hole (mass $M_{\mathrm{h}}$ ) with a distance of closest approach $b$, its velocity $\mathbf{V}$ at this point will

\title{
Real-time images of surface diffusion
}


4
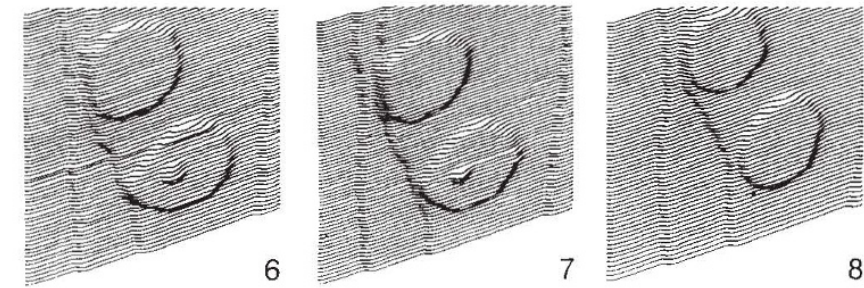

8

Scanning tunnelling microscopes (STMs) can be used to observe dynamic processes as they occur on surfaces. R.C. Jaklevic and L. Elie of the Ford Motor Company describe in a recent issue of Physical Review Letters $(60,120-123 ; 1988)$ how 'footprints' left in a gold surface by deliberate touches of the STM tip slowly fill in over a period of 2 hours. The micrographs above, taken at 16-minute intervals, represent an area $400 \times 400 \AA^{2}$. The step-height is $2.5 \AA$ - the height of a single gold atom - so that each footprint represents the loss of about 3,200 atoms. The holes fill in at a rate of only 6-9 atoms per minute, a rate unaffected by the scanning height of the STM tip or the size of tunnelling current used to detect the surface form. The authors tried to observe single atoms 'jumping' by leaving the tip at the edge of a crater, but failed possibly because of the rarity of such events, or because they are too rapid. (Courtesy of R.C. Jaklevic.) 